\title{
DISC REPLACEMENT IN LUMBAR SPINE
}

\author{
ARTROPLASTIA DE DISCO NA COLUNA LOMBAR \\ ARTROPLASTIA DE DISCO EN LA COLUMNA LUMBAR
}

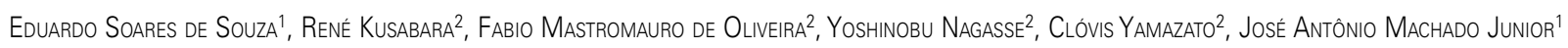

1. Spine Surgery Service of the Hospital Bandeirantes, São Paulo, SP, Brazil.

2. Sociedade Brasileira de Coluna, São Paulo, SP, Brazil.

\begin{abstract}
With the increase in the elderly population worldwide, the prevalence of degenerative diseases tends to grow, especially degenerative disc disease (DDD) in the lumbar spine. It is important to seek excellence treatments to ensure a better quality of life for patients. In this sense, the total disc replacement and the use of prostheses in the lumbar spine have been pointed out as alternative and promising treatments when compared to the standard treatment. The aim of this study is to show the results of total replacement of the intervertebral disc, through a review of the literature. The electronic search was performed in the PubMed databases, with the keywords "Total Disk Replacement", "Low Back Pain", and "Arthroplasty" in articles published as of 2012. We selected 16 articles. Level of Evidence: II. Type of study: Systematic review ${ }^{b}$ of Level II studies or Level I studies with inconsistent results.
\end{abstract}

Keywords: Total disc replacement; Low back pain; Arthroplasty.

\section{RESUMO}

Com o aumento da prevalência de idosos no mundo, a incidência de doenças degenerativas tende a crescer, especialmente a Doença degenerativa de disco (DDD) na coluna lombar. É importante buscar por tratamentos de excelência, para garantir melhor qualidade de vida aos pacientes. Nesse sentido, a substituição total do disco e o uso de próteses na coluna lombar tem sido apontados como tratamentos alternativos e promissores, quando comparado ao tratamento padrão. O objetivo deste artigo é mostrar os resultados da substituição total do disco intervertebral, por meio de uma revisão da literatura. Foi realizada a busca eletrônica nas bases de dados PubMed, a partir das palavras-chave: Substituição Total do Disco; Dor lombar; Artroplastia, publicados a partir de 2012. Foram selecionados um total de 16 artigos. Nível de Evidência: II. Tipo de estudo: Revisão sistemática ${ }^{b}$ de Estudos de nível II ou nível I com resultados discrepantes.

Descritores: Substituição total de disco; Dor lombar; Artroplastia.

\section{RESUMEN}

Con el aumento de la población de ancianos en todo el mundo, la prevalencia de enfermedades degenerativas tiende a crecer, especialmente la enfermedad degenerativa del disco (EDD) en la columna lumbar. Es importante buscar tratamientos de excelencia para garantizar una mejor calidad de vida a los pacientes. En este sentido, la sustitución del disco y el uso de prótesis en la columna lumbar han sido señalados como tratamientos alternativos y prometedores, en comparación con el tratamiento estándar. El objetivo de este estudio es mostrar los resultados de la sustitución total del disco intenvertebral, por medio de una revisión de la literatura. Se realizó la búsqueda electrónica en las bases de datos PubMed, con los descriptores "Reemplazo total de disco", "Dolor lumbar", "Artroplastia", en artículos publicados a partir de 2012. Se seleccionaron 16 estudios. Nivel de Evidencia: II. Tipo de estudio: Revisión sistemáticab de Estudios de nivel II o nivel I con resultados discrepantes.

Descriptores: Reemplazo total de disco; Dolor de la región lumbar; Artroplastia.

\section{INTRODUCTION}

Lumbar pain is one of the most common worldwide health complaints with an annual prevalence of $38 \%$. Its etiology is multifactorial and it can originate in the intervertebral discs, the facet joints, among other. ${ }^{1}$

Degenerative disc disease (DDD) stands out as the main risk factor for lumbar pain. This disease is a large health issue with a significant economic impact. $^{2}$

Lumbar fusion is the standard treatment; however, it is associated with rigidity and degeneration of the adjacent segment, which can lead to poor clinical outcomes and long-term complications ${ }^{2}$. Therefore, treatment is considered a therapeutic challenge. ${ }^{3}$

In this sense, total disc replacement (TDR) has emerged as an alternative to overcome the negative effects of spinal fusion. ${ }^{4}$

TDR has been receiving more attention in recent years. This procedure is designed to maintain the movement of the operated level and prevent degeneration of the adjacent segment. Despite this, there is still a lack of data about the best procedure to treat degenerative disc disease. ${ }^{2}$

In a study published in 2016, with the objective of verifying the main changes in the posterior paravertebral muscle following intervention with TDR or anterior lumbar fusion, the negative results of the restriction of movement in the posterior muscles, when compared to the preservation of movement, does not occur at a clinically relevant level. In this study, with a two-year follow-up, the restriction of movement resulting from fusion and the preservation of movement resulting from TDR caused small changes in the lumbar paravertebral musculature in relation to the atrophy of volume or degeneration of fat at the operated level, as well as in the upper adjacent segments. ${ }^{5}$

On the other hand, a systematic review of meta-analyses in 2017 , aimed at investigating the efficacy of TDR versus lumbar fusion in the treatment of DDD, indicated that current evidence suggests 
that TDR is a more efficient technique for the treatment of selected patients with DDD, and at worst, it is equally effective in the short term as lumbar fusion. ${ }^{2}$

Considering the importance of a better quality of life for patients with degenerative lumbar disease and the emergence of total disc replacement as a more advantageous surgical technique, the objective of this study was to identify updated information about total lumbar spine disc replacement.

\section{METHODS}

The objective of this study was to identify updated information about lumbar spine TDR based on the available literature. To this end, we conducted a search of articles in Pubmed using the following terms: total disc replacement, lumbar pain, arthroplasty. The following types of articles were selected: meta-analyses, systematic reviews, and randomized clinical studies with relevant clinical results, published starting in 2012 in journals with a consolidated WEBQUALIS score. A total of 16 articles were selected.

\section{Total disc replacement (TDR)}

Total disc replacement (TDR) in the lumbar spine has been used to relieve pain and discogenic dysfunction, while preserving the scope of segmental motion and restoring stability. ${ }^{1}$

Currently, the lumbar prostheses approved by the Food and Drug Administration (FDA) are the Charité Artificial Disc (DePuy Spine, Inc., Raynham, MA) approved in 2004 and the ProDisc Lumbar Disc Replacement (SYNTHES Spine, Inc., West Chester, PA) in 2006. In June, 2015, the activL Artificial Disc (Aesculap Implant Systems, LLC) received FDA approval for the TDR procedure. ${ }^{6}$

Despite promising results, the current use of TDR has decreased significantly since its introduction in mid-2000, for a variety of reasons: a higher cost than posterior fusion surgery, fear of the risk of late complications, and the incidence of reoperation. Other factors are the limited availability of evidence of its clinical efficacy and long-term safety. ${ }^{7}$

According to the authors, there are not sufficient data about long-term safety, so spine surgeons should be cautious about performing TDR on a large scale, a situation corroborated by Ding et al. ${ }^{2}$

In the United States, insurers have refused to reimburse surgeons who perform TDR procedures. The refusal is due to the fear of late complications, reoperations, and unknown secondary costs, a situation that has led to a drastic decline in the rates of TDR. ${ }^{8}$

\section{Selection criteria}

Lumbar TDR should be considered as a treatment option for patients who have had LDD for more than six months. The diagnosis should confirm the disc as the probable source of pain and the patient must not have facet joint degeneration, deformities, instabilities, or osteopenia/osteoporosis. ${ }^{6}$

First of all, patients with high pelvic incidence (greater than $65^{\circ}$ ) are prone to presenting arthritic changes in the facet joints, leading to less mobility and, thus, are not good candidates for TDR. Secondly, TDR is not intended to stabilize the spine and should not be used in patients with translational deformity, such as spondylolisthesis. Thirdly, this procedure is not suitable for patients with altered movements as a result of segmental autofusion (ankylosing spondylitis). Finally, TDR also requires stable fixation to the bone. Patients with poor bone quality can develop fixation failure and vertebral body fractures. ${ }^{6}$

The indication for this type of surgery should consider local parameters, such as the type of disc disease revealed in the magnetic resonance and the presence or absence of osteoarthritis of the facet joint. ${ }^{9}$

The type of preoperative sagittal curvature also should be taken into account. A meta-analysis was conducted in 2016 to verify whether the type of the sagittal spinal shape influences the clinical results of lumbar TDR. Five studies met the research criteria. They concluded that the type of sagittal curvature influences the clinical TDR results and that patients with curvature type four are associated with worse clinical results due to the higher rate of residual lumbar pain. Therefore, TDR should not be considered in these patients. A total of 80 patients with an average age of 41.7 years (27-56 years of age) and an average follow-up of 59.1 months (14-96 months) participated in this study. ${ }^{9}$

The amount of iatrogenic distraction, the preoperative disc height, and the positioning of the implant have been considered important for surgical success. Thus, Dreischarf et al. ${ }^{4}$ state that its effect on the postoperative range of motion and on facet load deserve additional discussion. In this sense, the authors developed a study to investigate the biomechanics of the L5-S1 motion segment following TDR.

\section{Surgical technique}

Regarding the surgical technique for total disc replacement, Salzmann et al. ${ }^{6}$ explain that the anatomical location of the great vessels is the basis for preference of the left side. The aorta (on the left) is not only easy to identify, but its wall is also more resistant compared to the lower vena cava (on the right). However, for access to L5-S1, especially in men, a right-side approach is preferable to avoid lesions of the superior hypogastric plexus (located in the anterior left part of the promontory) that can result in retrograde ejaculation. Subsequently, the disc is excised and the cartilaginous terminal plates are removed. Special care should be taken to preserve the integrity of the bone plates upon which the prosthesis will rest in order to help prevent subsidence. The TDR device should be implanted posteriorly, placing the center of rotation of the device in a favorable biomechanical position. Initial fixation is achieved through teeth (Charité, activL) or a keel (ProDisc-L) on the terminal endplates of the implant, which can be subsequently improved by osseointegration facilitated by the surface coating of the device. ${ }^{6}$

In 2011, Pimenta et al. described an alternative to the retroperitoneal approach technique that uses the XL-TDR device (NuVasive, Inc. San Diego, CA) and which demonstrated promising medium-term clinical results. ${ }^{10,11}$

The XL-TDR is a two-component metal-metal prosthesis consisting of two cobalt-chromium-molybdenum (CoCrMo) alloy plates coated with titanium plasma to facilitate bone growth. ${ }^{11}$

In 2017, a study reported an experience using the XL-STD device in a single location as an alternative to the retroperitoneal approach. The data analyzed was collected from 12 patients treated with $\mathrm{XL}$ TDR. The average follow-up was 27.5 months (18-48 months) and, according to the results, the patients improved significantly in terms of back $(74 \%)$ and leg $(50 \%)$ pain. Two patients had early luxation because of undersizing of the prosthesis. The same cutaneous incision was used to recover the XL-STD and to perform the intervertebral fusion and solid fusion was achieved within 12 months. They concluded that the XL-TDR via a lateral muscle division approach is a minimally invasive alternative to anterior retroperitoneal exposure for the preservation of movement. ${ }^{11}$

This situation is extremely important, since minimally invasive spine surgery is less aggressive and the small incisions and minimal dissection intrinsic to the surgery can reduce the risk of postoperative infection. Surgical infections are feared complications of spine surgery, with reported rates ranging from $0.09 \%$ to $16 \%$. They can lead to prolonged antibiotic therapy, prolonged hospitalization, repeated operations, and implant removal. ${ }^{12}$

In 2016, a literature review evaluated the incidence of surgical infections in minimally invasive spine surgery (MISS). Data from all patients who visited the hospital service between January 2007 and January 2017 and who underwent posterior spine surgery with a tubular retractor system and microscope were analyzed. The procedures performed included tubular discectomies, tubular decompression 
for spinal stenosis, and minimally invasive transforaminal lumbar interbody fusion (TLIF). A total of 1043 patients underwent 763 non-instrumented (discectomies, decompressions) and 280 instrumented (TLIF) surgeries. The average age was 52.2 years and the men:women ratio was 1.08:1. Three infections were encountered in the fusion surgeries (average hospitalization time of seven days). All three cases required cleaning and debriding of the wounds. One patient required total removal of the implant. The direct additional cost due to infection was US\$ 2678 (dollars) for 100 MISS-TLIFs. Surgical infection increased the per patient hospital expenses by 1.5 times. $^{12}$

\section{DEVICES}

\section{Prodisc II}

Zigler, Glenn, and Delamarter ${ }^{13}$ reported the results of a five-year prospective, randomized, multicenter study of degenerative changes of the adjacent level in patients with degenerative lumbar disease at a single level, treated with total lumbar disc replacement with ProDisc-L versus circumferential fusion. Degeneration at the adjacent level was characterized by a composite score including loss of disc height, sclerosis of the terminal plate, osteophytes, and spondylolisthesis. According to the results, after five years of follow-up, ALD changes were observed in $9.2 \%$ of the patients with TDR and in $28.6 \%$ of the patients with fusion. Among the patients without preoperative adjacent disease, ALD findings were evident after treatment in only $6.7 \%$ of the patients with TDR and $23.8 \%$ of the patients with fusion $(p=0.008)$. Surgery of the adjacent level that led to secondary surgery was reported for $1.9 \%$ of the patients with TDR and $4.0 \%$ of the patients with fusion $(p=0.6819)$. The patients with TDR had an average preoperative range of motion (ROM) of $7.3^{\circ}$ that had decreased slightly $\left(6.0^{\circ}\right)$ five years after treatment $(p=0.0198)$. From their results, the authors concluded that after five years, the ProDisc- $L$ has good postoperative range of motion and a significantly lower rate of ALD than in patients treated with circumferential fusion. ${ }^{13}$

In a series of prospective cases published in 2014, Siepe et al. reported the five to 10 year postoperative status of ProDisc II implants, considering the medium- and long-term clinical effectiveness, as well as the safety of the TDR in terms of perioperative complications and rates of reoperation. A total of 181 patients participated in the study, with an average follow up of 7.4 years, ranging from 5.0 to 10.8 years. According to the results, there were significant improvements in the $\mathrm{ODI}$ and VAS scores at all time points of postoperative follow-up ( $\mathrm{p}$ $<0.0001$ ). More than $86 \%$ of the patients were satisfied. The complication rate was $14.4 \%$ and the rate of reoperation was $16 \%$. The single-level TDR had fewer complications (11.9 vs. $27.6 \%, p=0.03$ ) and a higher satisfaction rate as compared to two-level cases. Taking the acceptable complication and reoperation rates into account, the authors concluded that the fear of excessive late complications after the implantation of ProDisc II is unwarranted. They concluded that ProDisc II implantation surgery is satisfactory in the medium and long terms. The safety of the procedure and surgical technique has been demonstrated through the complications and acceptable rates of reoperation Late or excessive complications or reoperations following the primary TDR procedure could not be confirmed with the current data. ${ }^{8}$

In 2016, another study to evaluate the long-term efficacy and safety of ProDisc II demonstrated that this device is associated with successful outcomes with a clinical success rate of $76.9 \%$ and a satisfaction rate of $87.2 \%$ in patients considered to be good candidates for TDR. However, patients with associated pathologies have suboptimal results and a high risk of revision surgery. Therefore, a rigorous patient selection process is mandatory for successful results. This study had a 120-month follow-up period and 54 patients categorized into two groups: Group A (Good candidates $=39$ patients) and Group B (Poor candidates $=15$ patients). The clinical success rate and the rate of satisfaction were significantly higher in Group A (76.9\% and 87.2\%, respectively) than in Group B $\left(40.0 \%\right.$ and $60.0 \%$, respectively). ${ }^{7}$

\section{Charité}

In a study published in 2016 by Guyer et al., the five-year results of the implants of two lumbar TDR devices were compared. This RCT included 190 patients who received Charité and 204 patients who received Kineflex-L. During the five-year follow-up, no significant differences were observed between the groups. The patients of both the Charité and Lineflex-L groups experienced significant clinical improvement as measured by ODI and VAS. The radiographic analysis showed that the segmental ROM in both groups decreased initially at three months, then increased up until 24 months, after which it remained stable. The reoperation rate in both groups was approximately $11 \%{ }^{14}$

\section{Activl}

The activL Artificial Disc is a biomimetic implant that incorporates various innovative characteristics not found in lumbar TDR of the previous generation. The device is aimed at accommodating a broader range of anatomical variations and it reproduces the kinematic patterns of the human lumbar spine with more precision. ${ }^{15}$

According to Garcia et al., ${ }^{1}$ the activL Artificial Disc is a modular prosthesis composed of an inferior plate of cobalt chromium alloy anchored to the terminal plate of the caudal vertebral body, an ultra high molecular weight polyethylene (UHMWPE) insert attached to the inferior component, and a superior cobalt chromium alloy component.

In 2016, Garcia et al. developed a prospective randomized study of one level to verify the safety and efficacy of the activL device in the treatment of TDR in people with DDD. According to the primary results, this device is safe and effective for the treatment of LDD in a single level in the L4-S1 interval. The study results show statistical non-inferiority of the experimental device as compared to the currently available FDA-approved control devices. The device being investigated also demonstrated superiority over the controls in terms of post-procedure range of motion. ${ }^{16}$

\section{CONCLUSIONS}

The total disc replacement technique is an alternative to lumbar fusion in the treatment of degenerative disc disease and has been considered promising for this pathology since it is associated with the preservation of movement, preventing disease at the adjacent level.

The main prostheses used in total lumbar disc replacement are the Charite Artificial Disc, ProDisc, and the activL Artificial Disc, all approved by the Food and Drug Administration in 2004, 2006, and 2015, respectively.

In spite of the evidence that proves the short-term benefits, more studies are needed to investigate the long-term safety and efficacy of total disc replacement

Considering the current evidence, TDR should be performed with caution at one level and in carefully selected patients.

Thanks to Dr. Rodrigo Amaral, of the Instituto Patologias da Coluna Vertebral (IPC), who shared his extensive experience in lumbar disc arthroplasty for the realization of this study.

All authors declare no potential conflict of interest related to this article.

CONTRIBUTION OF THE AUTHORS: Each author made significant individual contributions to this manuscript. ESS (0000-0001-9529-4731) ${ }^{\star}$ contributed to the development of the manuscript. ESS, RK(0000-0003-2673-7193)*, FMO(0000-0002-5217-1210)*, YN(0000-0003-0708-2961)* ${ }^{\star}, \mathrm{CY}(0000-0001-$ 7920-7634)* and JAMN(0000-0002-0028-9479)* contributed to the bibliographical review and survey. *ORCID (Open Researcher and Contributor ID). 


\section{REFERENCES}

1. Garcia RJ, Yue JJ, Blumenthal S, Coric D, Patel VV, Leary SP et al. Lumbar Total Disc Replacement for Discogenic Low Back Pain:Two-year Outcomes of the activL Multicenter Randomized Controlled IDE Clinical Trial. Spine 2015; 40(24): 1873-81.

2. Ding F, Jia Z, Zhao Z, Xie L, Gao X, Ma D, Liu M. Total disc replacement versus fusion for lumbar degenerative disc disease: a systematic review of overlapping meta-analyses. Eur Spine J. 2017; 26(3):806-815.

3. Andrieu K, Allainb J, Longis PM, Steib JP, Beaurain J, Delécrin J. Comparison between total disc replacement and hybrid construct at two lumbar levels with minimum follow-up of two years. OrthopTraumatol Surg Res. 2017;103(1): 39-43.

4. Dreischarf M, Schmidt H, Putzier M, Zander T. Biomechanics of the L5-S1 motion segment after total disc replacement - Influence of iatrogenic distraction, implant positioning and preoperative disc height on the range of motion and loading of facet joints. J Biomech. 2015; 48(12):3283-91.

5. Strube P, Putzier M, Streitparth FMD, Eike K, Hartwig T. Postoperative posterior lum bar muscle changes and their relationship to segmental motion preservation or restriction: a randomized prospective study. Journal of Neurosurgery: Spine. 2016; 24(1):25-31.

6. Salzmann SN, Plais N, Shue J, Girardi FP. Lumbar disc replacement surgery-successes and obstacles to widespread adoption. Curr Rev Musculoskelet Med. 2017;10(2):153-9.

7. Park SJ1, Lee CS, Chung SS, Lee KH, Kim WS, Lee JY. Long-Term Outcomes Following Lumbar Total Disc Replacement Using ProDisc-II: Average 10-Year Follow-Up at a Single Institute. Spine (Phila Pa 1976). 2016;41(11):971-7.

8. Siepe CJ, Heider F, Wiechert K, HitzI W, Ishak B, Mayer MH2. Mid- to long-term results of total lumbar disc replacement: a prospective analysis with 5- to 10-year follow-up. Spine J. 2014;14(8):1417-31.

9. Laouissat F, Scemama C, Delécrin J. Does the type of sagittal spinal shape influence the clinical results of lumbar disc arthroplasty?. Orthop Traumatol Surg Res. 2016; 102(6):765-8.

10. Pimenta L, Oliveira L, Schaffa T, Coutinho E, Marchi L. Lumbar total disc replacement from an extreme lateral approach: clinical experience with a minimum of 2 years' follow-up. J Neurosurg Spine. 2011:14(1):38-45.

11. Malham GM, Parker RM. Early experience with lateral lumbar total disc replacement: Utility, complications and revision strategie S J Clin Neurosci. 2017;39:176-83.

12. Kulkarni AG, Patel RS,Dutta S. Does Minimally Invasive Spine Surgery Minimize Surgical Site Infections?. Asian Spine J. 2016; 10(6): 1000-6.

13. Zigler JE, Glenn J, Delamarter RB. Five-year adjacent-level degenerative changes in patients with single-level disease treated using lumbar total disc replacement with ProDisc-L versus circumferential fusion. J Neurosurg Spine. 2012;17(6):504-11.

14. Guyer R, Pettine, K, Roh JS, Thomas A, Coric D, McAfee P. Five-Year Follow-Up of a Prospective, Randomized Trial Comparing Two Lumbar Total Disc Replacements. Spine 2016; 41(1): 3-8.

15. Yue JJ, Garcia R Jr, Miller LE. The activL(®) Artificial Disc: a next-generation motion-preserving implant for chronic lumbar discogenic pain. Med Devices (Auckl). 2016; 9:75-84.

16. Garcia J, Yue JJ, Blumenthal SL, Coric D, Patel VV. 153 - LumbarTotal Disc Replacement for Discogenic Low Back Pain: Primary Powered Endpoint Outcomes of The ActivL® IDE ClinicalTrial. The Spine Journal . 2016; 16(10): S260-S260. 